\title{
ABNORMAL BONE TURNOVER IN INDIVIDUALS WITH LOW SERUM ALKALINE PHOSPHATASE
}

Laura López-Delgado ${ }^{1}$, Leyre Riancho-Zarrabeitia*2, Maria T. García-Unzueta ${ }^{3}$, Jair A. Tenorio ${ }^{4}$, Marta García-Hoyos ${ }^{1}$, Pablo Lapunzina ${ }^{4}$, Carmen Valero ${ }^{1}$ and José A. Riancho ${ }^{1}$

1. Service of Internal Medicine, Hospital U.M. Valdecilla, University of Cantabria, IDIVAL. Santander, Spain.

2. Service of Rheumatology. Hospital Sierrallana, Torrelavega, Spain

3. Service of Clinical Biochemistry, Hospital U.M. Valdecilla, University of Cantabria, IDIVAL. Santander, Spain.

4. Institute of Medical and Molecular Genetics (INGEMM), Hospital Universitario La Paz. IdiPAZ. Universidad Autónoma de Madrid and CIBERER, ISCIII. Madrid, Spain.

(*) Both authors contributed equally to this study

\section{Correspondence}

José A. Riancho

Service of Internal Medicine, Hospital U.M. Valdecilla,

University of Cantabria

Av Valdecilla SN

39008 Santander, Spain

Email: rianchoj@unican.es 


\section{MINI-ABSTRACT}

We evaluated bone mineral density (BMD), bone microarchitecture and bone turnover markers in patients with low serum levels of alkaline phosphatase. Our results show that these patients have low bone remodeling even in the absence of BMD abnormalities, thus supporting the recommendation of avoiding antiresorptives such as bisphosphonates in these subjects. 


\section{ABSTRACT}

The clinical spectrum of hypophosphatasia (HPP) is broad and variable within families. Along severe infantile forms, adult forms with mild manifestations may be incidentally discovered by the presence of low alkaline phosphatase (ALP) activity in serum. However, it is still unclear whether individuals with persistently low levels of ALP, in the absence of overt manifestations of HPP, have subclinical abnormalities of bone remodeling or bone mass. The aim of this study was to obtain a better understanding of the skeletal phenotype of adults with low ALP by analyzing bone mineral density (BMD), bone microarchitecture (Trabecular bone score, TBS), and bone turnover markers (P1NP and ß-crosslaps). We studied 42 individuals with persistently low serum ALP. They showed lower levels of P1NP (31.4 \pm 13.7 versus $48.9 \pm 24.4 \mathrm{ng} / \mathrm{ml} ; \mathrm{p}=0.0002)$ and $\beta$-crosslaps $(0.21 \pm 0.17$ versus $0.34 \pm 0.22 \mathrm{ng} / \mathrm{ml}, \mathrm{p}=0.0015)$ than individuals in the control group. There were no significant differences in BMD, bone mineral content or TBS. These data suggest that individuals with hypophosphatasemia have an overall reduction of bone turnover, even in the absence of overt manifestations of HPP or low BMD.

KEYWORDS: hypophosphatasia, hypophosphatasemia, bone turnover markers, bone mineral density 


\section{INTRODUCTION}

Hypophosphatasia (HPP) is a rare genetic condition caused by loss-of-function mutations of the gene that encodes the tissue nonspecitific alkaline phosphatase (ALPL or TNSALP) located on chromosome 1p36.12 (1). In humans, there are four alkaline phosphatase (ALP) isoenzymes which are encoded by separate genes. Three of them are expressed in a tissue specific distribution and produce the intestinal, placental, and germ cell (placental-like) ALP. The fourth gene (ALPL) encodes the tissuenonspecific ALP (2) and is mainly expressed in bone, liver and kidneys. In HPP, deficient phosphohydrolase activity leads to extracellular accumulation of its natural substrates, including inorganic pyrophosphate (PPi), a potent inhibitor of hydroxyapatite cristal formation and propagation. The accumulation of extracelular PPi blocks mineralization and the patients consequently develop abnormalities of tissues with a mineralized matrix, including teeth and bone (3). More than 300 mutations of ALPL have been described and are included in the ALPL gene mutation database (4). Most of them are missense mutations and the inheritance pattern can be recessive or dominant.

The clinical spectrum of HPP is broad and variable within families. Along severe infantile forms, there are adult forms with mild manifestations that may be incidentally discovered by the presence of Iow ALP activity in serum. In a previous report (5) we studied 42 individuals with persistent and unexplained low levels of ALP and found that $50 \%$ of them carried a mutation in the ALPL coding region. Although clinical manifestations were usually mild, in about $50 \%$ of cases the enzymatic activity was low enough to cause the accumulation of phosphorylated substrates. It is still unclear whether individuals with persistently low levels of ALP, in the absence of overt manifestations of HPP, present subclinical abnormalities of bone remodeling or bone mass. Nevertheless, in the previous study we unexpectedly found low levels of immunoreactive bone ALP (BALP) in patients with low ALP. BALP was determined by EIA, a method that measures the protein concentration, not the enzymatic activity. Therefore, that result suggested an abnormal bone remodeling in those individuals. Hence, the aim of this study was to obtain 
a better understanding of the skeletal phenotype of adults with low ALP by analyzing bone mineral

\section{METHODS}

We studied 42 unrelated individuals (10 men, 32 women) with persistently low levels of ALP not related to drug therapy or other secondary cause. All had at least one determination below $27 \mathrm{U} / \mathrm{I}$ and none within the normal range $(40-160 \mathrm{U} / \mathrm{I})$. Details about the recruitment have been previously reported (5). These included acute diseases, malnutrition, celiac disease, hypothyroidism, parathyroid disease, cancer and renal failure, as well as treatment with antiresorptives or glucocorticoids. Most subjects were asymptomatic or had mild nonspecific ailments. They did not receive anti-resorptives or other drugs known to interfere with bone metabolism. The comparison group included 45 healthy controls (10 men, 35 women).

The serum levels of bone turnover markers P1NP and B-crosslaps were measured with automatized chemiluminiscence assays (IDS-iSYS Multi-Discipline Automated Analyzer) in the morning after overnight fasting. Sensitivity was $1 \mathrm{ng} / \mathrm{ml}$ and $0.033 \mathrm{ng} / \mathrm{ml}$ respectively. The intra-assay coefficient of variation (CV) was 2.9 and $4.6 \%$, respectively; and the interassay CV was 3.2 and $6.2 \%$, respectively. To avoid batch-related biases, similar numbers of samples from the patient and control groups were included in each run.

Lumbar spine (L1-L4) and hip BMD were also evaluated by DXA (Hologic QDR 4500, Waltham, MA) in 20 individuals with low ALP who consented to be studied. TBS was obtained from DXA scans with the use of software v2.1.

Comparisons between groups were analyzed by Mann-Whitney $\mathrm{U}$ and Spearman correlation tests. All tests were 2 -tailed and $p$-values less than 0.05 were considered as statistically significant. 


\section{RESULTS}

Remarkably, patients with low levels of ALP presented lower levels of P1NP and B-crosslaps than individuals in the control group (table 1, and supplementary table S1). The difference was independent of age, and consequently it was maintained in the age-adjusted analysis. ß-crosslaps and P1NP levels were positively correlated $(r=0.64 ; p<0.001)$. ß-crosslaps levels were also inversely correlated with serum calcium ( $r=-0.41, p=0.007$ ). However, there were no significant correlations between serum $\beta$-crosslaps or P1NP and serum levels of ALP, phosphorus, or pyridoxal phosphate, nor with urine levels of phosphoetanolamine.

ALPL mutations were present in 21 patients ( 20 heterozygotes and 1 homozygote). The levels of ALP were slightly higher in the group without mutation, in comparison with the group with mutations detected ( $30 \pm 6$ versus $25 \pm 6 \mathrm{U} / \mathrm{l} ; \mathrm{p}=0.014)$, both lower than the mean level in controls $(64 \pm 18 \mathrm{U} / \mathrm{l}$; $\mathrm{p}<0.0001)$. The presence of an ALPL mutation was not associated with significant differences in BTM levels (figure 1).

We also evaluated BMD in 20 of these individuals with low ALP (6 men 14 women; age $66 \pm 16 \mathrm{yr}$ ), 10 of which had an ALPL mutated allele. The results were compared with 80 healthy controls ( 30 men, 50 females; age $54 \pm 14 \mathrm{yr}$ ). Both groups showed similar bone mineral content (BMC) and BMD at the spine and the hip. Lumbar Spine BMD was $1.028 \pm 0.182$ in cases and $0.955 \pm 0.163 \mathrm{~g} / \mathrm{cm}^{2}$ in controls $(p=0.09)$; femoral neck BMD was $0.817 \pm 0.156$ in cases and $0.775 \pm 0.120 \mathrm{~g} / \mathrm{cm}^{2}$ in controls $(p=0.20)$; total hip BMD was 0.9400 .168 and $0.919 \pm 0.139$ in cases and controls, respectively $(p=0.57)$. In line with these results, there were no significant differences in the age-adjusted BMD between patients with and without ALPL mutations. The mean trabecular bone score (TBS) was $1.41 \pm 0.09$ in patients and $1.37 \pm 0.14$ in controls $(p=0.50)$ in all cases it was higher than 1.20 , the lower limit of the normal range.

\section{DISCUSSION}

The incidence of severe HPP is approximately 1 in 300,000 in Europe (6). However, the clinical spectrum of HPP is widely variable, with severe forms usually manifesting during perinatal and/or 
infantile periods, while mild forms are sometimes only diagnosed in adulthood or remain undiagnosed.

Thus, recent studies suggest that less severe forms observed in adults might be substantially more frequent. Mornet et al (7) estimated the prevalence of moderate forms of HPP to be 1/6730.

The clinical significance of persistently low serum levels of ALP in patients who otherwise do not have overt manifestations of HPP is unclear, for those individuals may be asymptomatic or have just mild and nonspecific symptoms. Thus, it may be difficult to establish a causal relationship between such ailments and the enzymatic defect. However, it has been suggested that those patients may be at risk of developing complications if treated with drugs interfering with bone turnover, including the development of atypical femoral fractures related to anti-resorptive therapy (8).

Thus, the aim of this study was to investigate if patients with low ALP levels but without overt HPP have an abnormal bone homeostasis. We found that patients with persistently low ALP indeed have a reduced bone turnover, as assessed by the bone formation marker P1NP and the bone resorption marker $\beta$-crosslaps. In other words, they have a global alteration of bone metabolism and not just a reduced ALP activity. The lower turnover found in this study is consistent with the observations in patients with HPP. Most patients with HPP appear to have, along the delayed mineralization with osteoid accumulation, low numbers of osteoblasts and osteoclasts, and absence of changes of secondary hyperparathyroidism in patients. Similar findings were reported in ALPL knock-out mice $(9,10,11,12)$. However, patient heterogeneity appears to exist not only from the clinical point of view, but also from the histological perspective, and high osteoblast numbers have been reported in a few patients (13).

It is worth mentioning that these abnormalities were present in heterozygous patients, which is consistent with the results of genetic models. In fact, studies with ALPL knock-out mice showed that even heterozygous osteoblasts had an impaired ability to form a mineralized matrix (14). We did not find clear biochemical differences between patients with hypophosphatasemia with mutations of the ALP gene and those with hypophosphatasemia but no evidence of gene mutations. However, since we only sequenced the coding region of the gene, we cannot exclude the possibility that those patients had mutations in regulatory regions of the ALPL gene, or in other trans-acting regulators of ALP activity. 
The mechanisms linking low ALP expression and reduced bone turnover are unclear. It could be

speculated that the accumulation of osteoid with delayed mineralization could tend to decrease the differentiation of osteoblast precursors, through some unidentified feedback mechanisms. This could be consistent with the results of bone biopsies in patients with HPP. The intriguing results of a recent study showing that an anti-sclerostin monoclonal antibody enhances bone formation markers in patients with HPP raise the possibility that Wnt signaling may be involved (15). ALP is usually regarded as a product of the osteoblastic lineage. However, other cells may also express the enzyme within the bone microenvironment. In particular, ALP activity is also expressed in preosteoclasts. Quite interesting, enzyme activity is concentrated in the basolateral membrane in mature resorbing osteoclasts, suggesting that ALP might also play some role in bone resorption (16). However, the role of ALP in bone resorption remains merely speculative at the present.

Despite the decreased bone turnover, we did not find differences in BMD or TBS between patients and controls, which is consistent with the mostly normal BMD values found in other series of adult patients with HPP (17).

In 2012, Sutton et al (8) reported the first case of BP exposure preceding atypical femoral fractures (AFFs) in adult HPP. Other authors such as McKiernan et al (18) have also recommended against the use of biphosphonates (BP) in patients with suspected HPP. Long term treatment with BPs has been associated with AFFs (19). BPs are analogues of PPi and can suppress bone turnover but also deactivate ALP (20). Because BPs are analogs of PPi, it has been speculated that HPP patients may be sensitized to the effects of BPs. Despite the absence of obvious BMD abnormalities, our findings of low bone remodeling in patients with low levels of ALP, even in the absence of overt skeletal manifestations, support the recommendation of avoiding antiresorptives such as bisphosphonates in these subjects.

Our study has some limitations, such as the smallnumber of individuals with DXA data, and the absence of histological analysis. Nevertheless, these results strongly suggest that patients with heterozygous mutations of the ALPL gene and other with persistent hypophosphatasemia have low bone 
remodelling even though they do not present evident clinical skeletal alterations or decreased BMD. This might render them more susceptible to anti-resorptive-related adverse effects.

Authors' roles: Study design: JAR. Data collection, BMD analysis, biochemical analyses: LLD,LRZ, CV, MGH, MGU. Genetic analysis: PL, JAT. Data analysis: LLD, JAR. Drafting manuscript: LLD, LRZ, JAR. Critical revising manuscript content: all authors. Approving final version of manuscript: all authors. JAR takes responsibility for the integrity of the data analysis. 


\section{REFERENCES}

1. Millan JL and Whyte MP. Alkaline phosphatase and hypophosphatasia. Calcif Tissue Int. 2015;98: 398-416

2. Whyte MP. Physiological role of alkaline phosphatase explored in hypophosphatasia. Ann N Y Acad Sci. 2010;1192: 190-200.

3. Whyte MP. Hypophosphatasia: Enzyme Replacement Therapy Brings New Opportunities and New Challenges. J Bone Miner Res. 2017; 32: 667-675

4. Silvent J, Gasse B, Mornet E et al. Molecular evolution of the tissue-nonspecific alkaline phosphatase allows prediction and validation of missense mutations responsible for hypophosphatasia. J Biol Chem. 2014; 289: 24168-24179.

5. Riancho-Zarrabeitia L, Garcia-Unzueta M, Tenorio JA et al. Clinical, biochemical and genetic spectrum of low alkaline phosphatase levels in adults. Eur J Intern Med. 2016;29: 40-45.

6. Fraser, D. Hypophosphatasia. Am J Med. 1957; 22: 730-746

7. Mornet E, Yvard A, Taillandier A et al. A molecular-based estimation of the prevalence of hypophosphatasia in the European population. Ann Hum Genet. 2011;75: 439-445.

8. Sutton RA, Mumm S, Coburn SP et al. Atypical femoral fractures during bisphosphonate exposure in adult hypophosphatasia. J Bone Miner Res. 2012; 27: 987-94.

9. MacDonald AM, Shanks RA. Hypophosphatasia. Arch Dis Child. 1957; 32: 304-10

10. Whyte MP, Teitelbaum SL, Murphy WA. Adult hypophosphatasia. Clinical, laboratory, and genetic investigation of a large kindred with review of the literature. Medicine (Baltimore). 1979; 58: 329-47.

11. Weinstein RS, Whyte MP. Fifty-year follow-up of hypophosphatasia. Arch Intern Med. 1981;141: 1720-1

12. Fedde KN, Blair L, Silverstein J et al. Alkaline phosphatase knock-out mice recapitulate the metabolic and skeletal defects of infantile hypophosphatasia. J Bone Miner Res. 1999;14: 2015-26

13. Barvencik F, Beil FT, Gebauer M et al. Skeletal mineralization defects in adult hypophosphatasia-a clinical and histological analysis. Osteoporos Int. 2011;22: 2667-75.

14. Wennberg $C$, Hessle L, Lundberg P et al. Functional characterization of osteoblasts and osteoclasts from alkaline phosphatase knockout mice. J Bone Miner Res. 2000;15: 1879-88.

15. Seefried L, Baumann J, Hemsley S et al. Efficacy of anti-sclerostin monoclonal antibody BPS804 in adult patients with hypophosphatasia. J Clin Invest. 2017;127: 2148-2158.

16. Nakamura $\mathrm{Y}$, Tanaka $\mathrm{T}$, Wakimoto $\mathrm{Y}$ et al. Alkaline phosphatase activity in the osteoclasts induced by experimental tooth movement. J Electron Microsc (Tokyo). 1991; 40: 403-6.

17. Schmidt $\mathrm{T}$, Mussawy $\mathrm{H}$, Rolvien $\mathrm{T}$ et al. Clinical, radiographic and biochemical characteristics of adult hypophosphatasia. Osteoporos Int. 2017; 28: 2653-2662.

18. McKiernan FE, Berg RL, Fuehrer J. Clinical and radiographic findings in adults with persistent hypophosphatasemia. J Bone Miner Res. 2014;29: 1651-60.

19. Odvina CV, Levy $S$, Rao $S$, et al. Unusual mid-shaft fractures during long-term bisphosphonate therapy. Clin Endocrinol (Oxf). 2010;72: 161-168

20. Vaisman DN, McCarthy AD, Cortizo AM. Bone-specific alkaline phosphatase activity is inhibited by bisphosphonates: role of divalent cations. Biol Trace Elem Res. 2005;104: 131-40 
Table 1. Main characteristics and bone turnover markers in cases and controls (mean $\pm S D$ )

\begin{tabular}{|l|c|c|r|}
\hline & $\begin{array}{c}\text { Low-ALP cases } \\
(\mathbf{n}=\mathbf{4 2})\end{array}$ & $\begin{array}{c}\text { Controls } \\
(\mathbf{n}=\mathbf{4 5})\end{array}$ & p-value \\
\hline Age (yr) & $48 \pm 14$ & $47 \pm 18$ & $\mathrm{~ns}$ \\
\hline Sex & $10 \mathrm{M}, 32 \mathrm{~F}$ & $10 \mathrm{M}, 35 \mathrm{~F}$ & $\mathrm{~ns}$ \\
\hline Height (cm) & $163 \pm 9$ & $163 \pm 9$ & $\mathrm{~ns}$ \\
\hline Weight (kg) & $70 \pm 17$ & $66 \pm 14$ & $\mathrm{~ns}$ \\
\hline P1NP (ng/ml) & $31.4 \pm 13.7$ & $48.9 \pm 24.4$ & 0.0002 \\
\hline Crosslaps (ng/ml) & $0.21 \pm 0.17$ & $0.34 \pm 0.22$ & 0.0015 \\
\hline
\end{tabular}




\section{FIGURE LEGEND}

Fig 1. Bone turnover markers in individuals with low ALP levels, without (dashed bars) or with mutations (grey solid bars) of the ALPL gene, in comparison with controls (black solid bars). 
(ן/[6u) sdejssodj-g

옹

蒙
$\square$
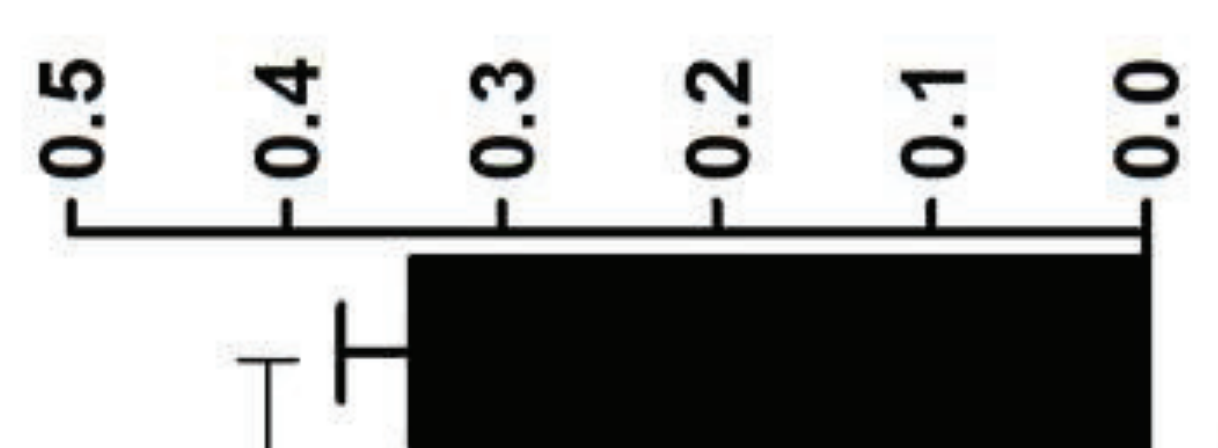

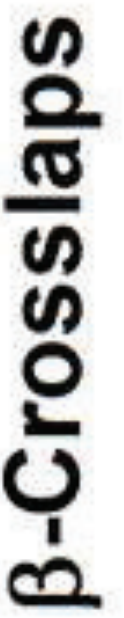

节

\&

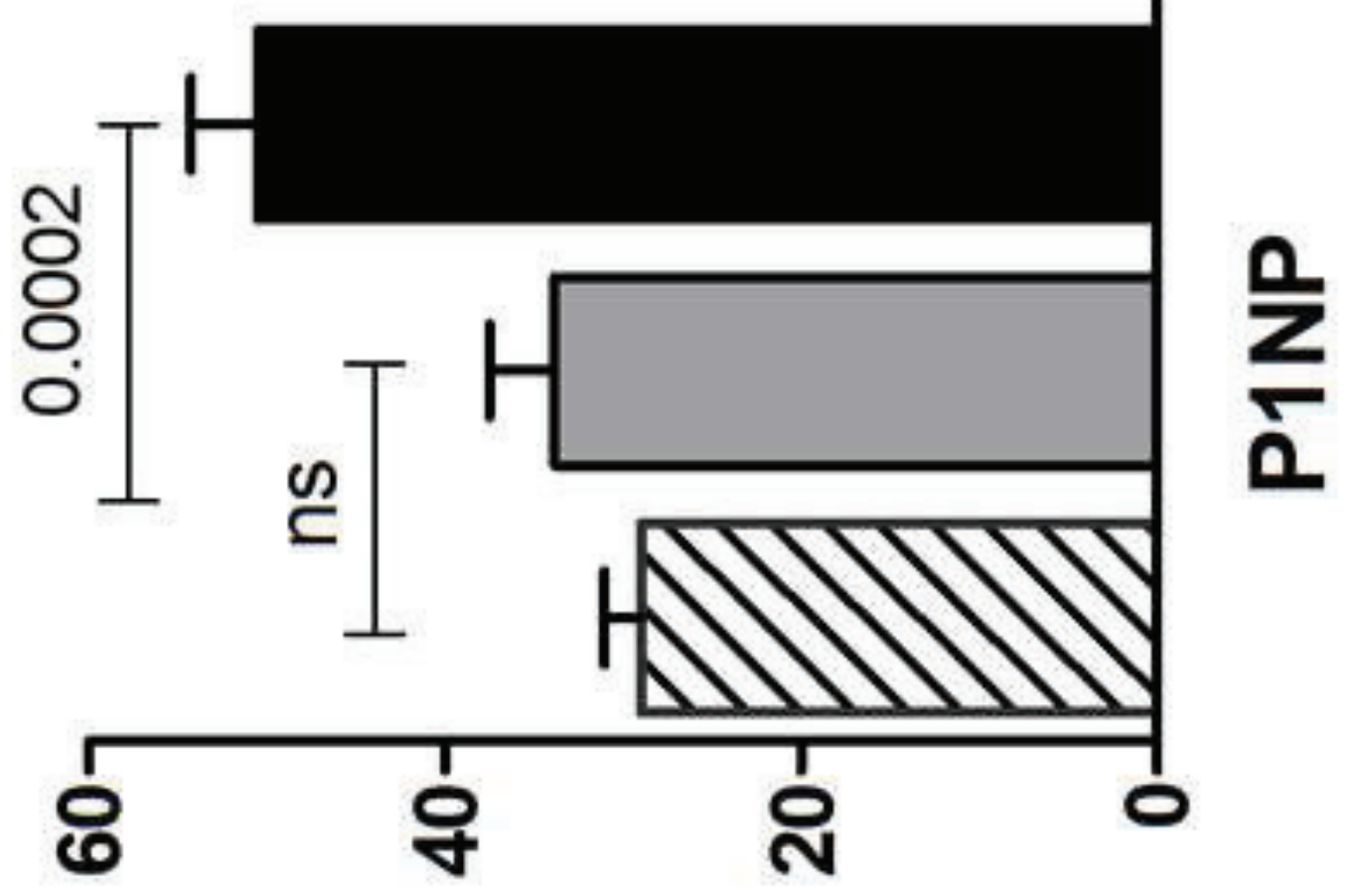

(IJ/6u) dNLd 


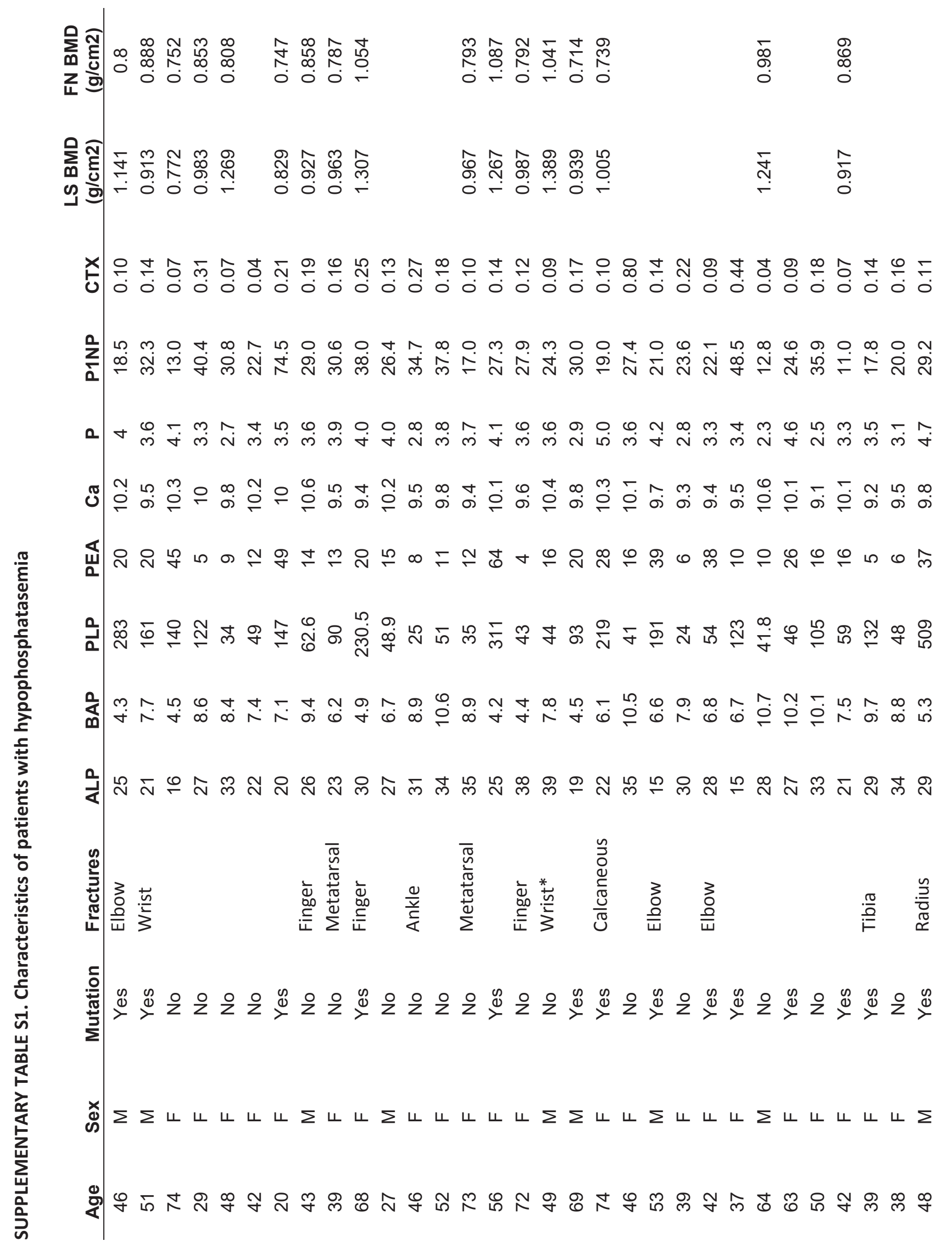




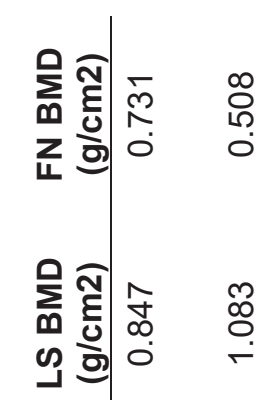

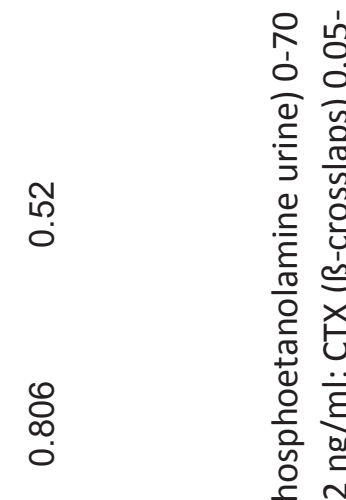

들 ㄱำ

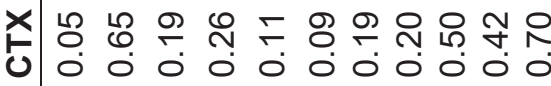

㟧

$\stackrel{2}{\overline{0}}$

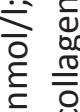

约

ż

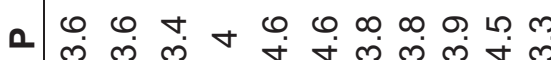

긍

๗ั)

ఖ

茎范

응 음

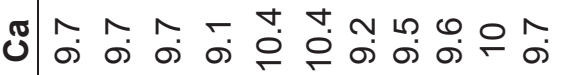

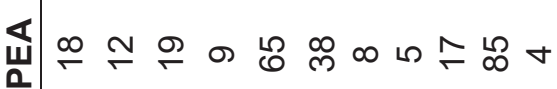

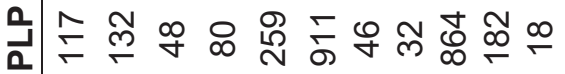

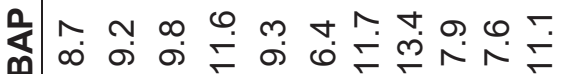

บ $\approx$ ฟ

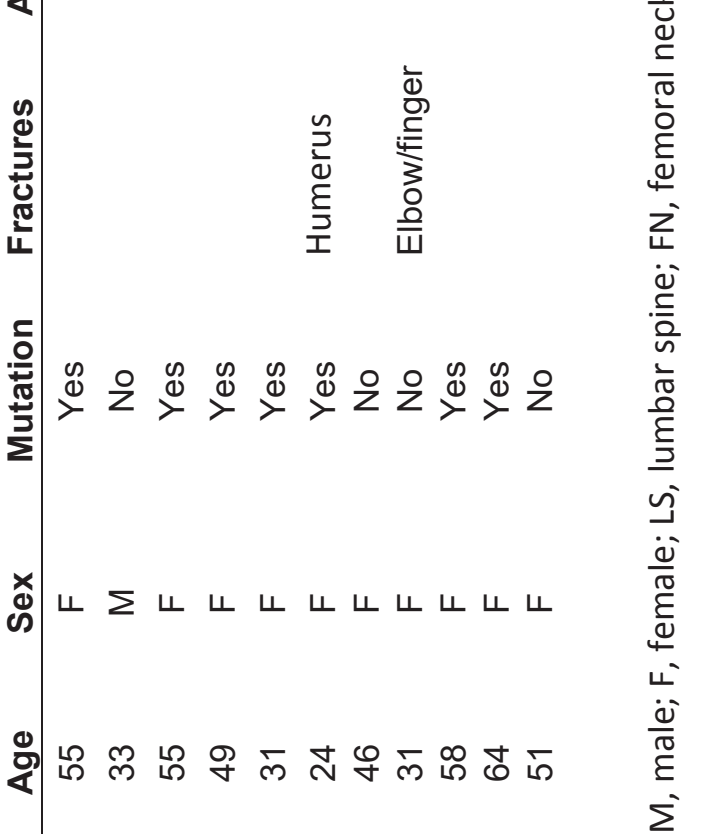

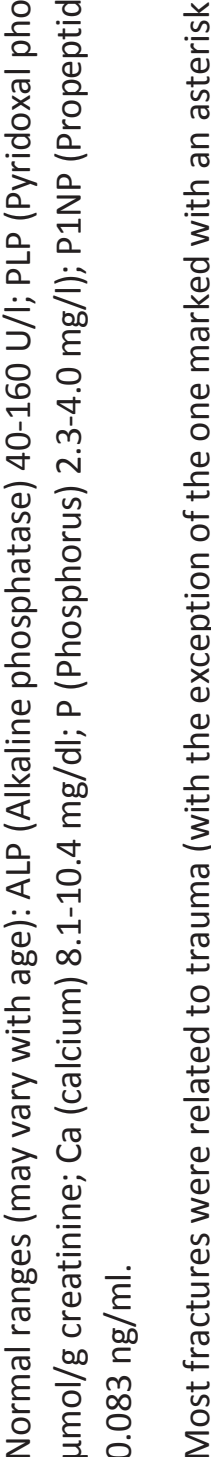

